\title{
Impedance model of trap states for characterization of organic semiconductor devices
}

\author{
L. Burtone, ${ }^{\text {a) }}$ D. Ray, K. Leo, and M. Riede \\ Institut für Angewandte Photophysik, Technische Universität Dresden, Dresden 01062, Germany
}

(Received 7 February 2012; accepted 15 February 2012; published online 20 March 2012)

\begin{abstract}
An equivalent circuit to characterize energy distribution of trap states present in organic semiconductors by impedance spectroscopy is proposed. We analyze the impedance spectra of a small-molecule organic solar cell and observe the contribution of trap states at low frequencies. Starting from previously reported equivalent circuits and a theoretical model based on the integration of a general traps distribution, we develop an equivalent circuit, which is able to describe the energetic distribution of trap states typically observed in organic semiconductors. The experimental data can be reproduced by our equivalent circuit, and we estimate a density of trap states in a Zn-phthalocyanine:C60 bulk heterojunction to be about $1.9 \pm 0.6 \times 10^{16} \mathrm{~cm}^{-3} \mathrm{eV}^{-1}$. (C) 2012 American Institute of Physics. [http://dx.doi.org/10.1063/1.3693545]
\end{abstract}

\section{INTRODUCTION}

During the past decade, organic electronic devices have attracted increasing interest, due to applications ranging from light-emitting devices to solar cells. ${ }^{1,2}$ Recently, organic solar cells have seen a steady increase in device performance, and power conversion efficiency above $8 \%$ have been reported. ${ }^{3-5}$ For small molecule organic solar cells (SMOSC), this has been possible by the introduction of the p-i-n architecture with doped hole transport layer (HTL) and electron transport layer (ETL), which brought several electrical and optical advantages. ${ }^{6,7}$

Further improvement in the performance of $p-i-n$ devices requires a better understanding of the doping mechanism and electrical properties of the devices. In the past, impedance spectroscopy (IS) has been extensively used to characterize inorganic semiconductor devices, ${ }^{8,9}$ and it is attracting a growing interest also for organic semiconductors. ${ }^{10-12}$ Even though the measurement technique is the same, many differences arise with respect to inorganic semiconductors when analyzing the frequency response of organic materials, due to the different charge carrier transport mechanisms and energetic distribution of states. A better understanding of the impedance spectra is therefore necessary to correctly interpret the measured data in the case of organic semiconductors.

In order to describe any electronic device, it is important to investigate the presence of trap states. They can modify the electric field inside the device, considerably affecting charge generation and transport and, consequently, the solar cell efficiency. ${ }^{13-15}$ The presence of intra-gap states, especially at the interfaces, has been widely reported in the past, mostly for polymeric solar cells. ${ }^{16,17}$ By analyzing impedance spectra, we observe trap states in small molecule solar cells.

In order to characterize them, we propose a new equivalent circuit derived from previously reported theoretical

${ }^{\text {a)} E l e c t r o n i c ~ m a i l: ~ l o r e n z o . b u r t o n e @ i a p p . d e . ~}$ models. ${ }^{18-20}$ Since we are interested in the description of a distribution of intra-gap states, we consider only the impedance spectra measured in the dark. The processes that govern the charge transport in the device can be described by varying the bias voltage. The effect of light on the impedance spectra of an organic solar cell is out of the scope of this paper and represents an outlook for future work.

\section{EXPERIMENTAL}

The investigated devices are typical solar cells with p-i-n structure $^{21}$ built on a glass/indium tin oxide (ITO) substrate (Thin Film Devices, USA). The active layer is composed of a zinc-phthalocyanine (ZnPc, TCI Europe)/C60 (Bucky, USA) bulk heterojunction with a ZnPc:C60 volume ratio of 1:2.3. Both $\mathrm{ZnPc}$ and $\mathrm{C} 60$ were purified at least twice by vacuum gradient sublimation. The density values are estimated by $\mathrm{x}$ ray reflectivity measurements to be $1.55 \pm 0.05 \mathrm{~g} / \mathrm{cm}^{-3}$ and $1.63 \pm 0.05 \mathrm{~g} / \mathrm{cm}^{-3}$ for ZnPc and C60, respectively. The p-i-n structure is obtained by adding doped transport layers between the active region and the metal contacts (Fig. 1). The resulting structure is: ITO/5 wt. \% p-doped $\mathrm{N}, \mathrm{N}, \mathrm{N}^{\prime}, \mathrm{N}^{\prime}-$ tetrakis(4-methoxyphenyl)-benzidine (MeO-TPD, Sensient) $(50 \mathrm{~nm}) / \mathrm{ZnPc}: \mathrm{C} 60(48 \mathrm{~nm}) / 3$ wt. \% n-doped C60 (50 nm)/Al $(100 \mathrm{~nm})$. For doping MeO-TPD, we use the p-dopant C60F36 (Ref. 22) from MTR Ltd., and for n-doping C60, we use W2(hpp)4 (Ref. 23) from Novaled AG (Dresden, Germany). The active area is defined as the geometric overlap between ITO and $\mathrm{Al}$ and results to be $6.44 \mathrm{~mm}^{2}$.

Our measurements are performed in the dark at room temperature with an Autolab PG- STAT302 N. The probe signal has an amplitude of $10 \mathrm{mV}(\mathrm{rms})$, and the frequency is varied from $10 \mathrm{~Hz}$ to $1 \mathrm{MHz}$.

\section{RESULTS DISCUSSION}

\section{A. Current-voltage characteristic}

The current-voltage characteristic of the solar cells is presented in Fig. 2 in the dark (open symbols) and under 


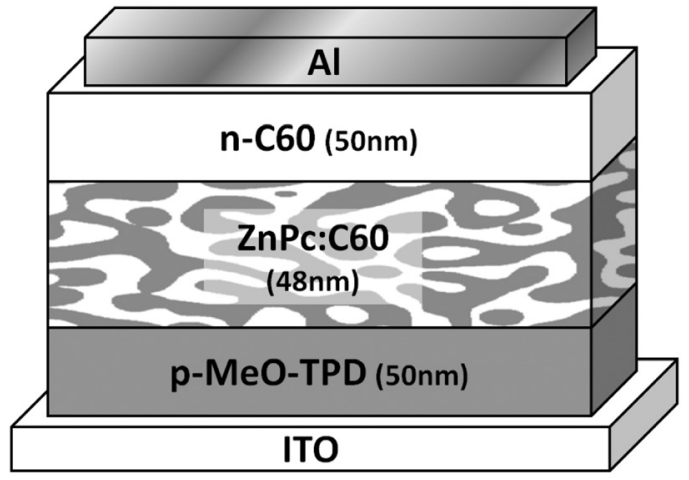

FIG. 1. Structure of the devices measured. The solar cells have a p-i-n structure with doped transport layers and a ZnPc:C60 bulk heterojunction as photo-active layer.

$100 \mathrm{~mW} / \mathrm{cm}^{2}$ AM1.5 illumination, as measured with respect to a reference standard silicon photodiode (solid symbols). In the inset, the J-V curve in dark is shown in a semilogarithmic scale. The devices have an efficiency of around $1.8 \%$ without considering any mismatch correction between the reference photodiode and the solar cell response.

Impedance spectroscopy (IS) is an established technique to identify and distinguish the dynamics of the different processes that govern the electrical response of the device. ${ }^{24}$ In order to have a sensitive IS characterization, a large rectification of the solar cells, which shows very low reverse currents in dark conditions, is necessary. The low reverse currents in the solar cell structure means that the device is dominantly capacitive under such conditions; therefore, the capacitance can be measured to a high degree of accuracy.

\section{B. Impedance spectra}

The results are presented in terms of absolute value and phase of the complex impedance function in the Bode plot. In Fig. 3(a) and Fig. 3(b), the modulus and the phase of the measured impedance are shown. The bias voltage is changed from $0 \mathrm{~V}$ to $0.6 \mathrm{~V}$, i.e., forward bias. In reverse bias, no significant change, with respect to the spectra at $0 \mathrm{~V}$, is visible.

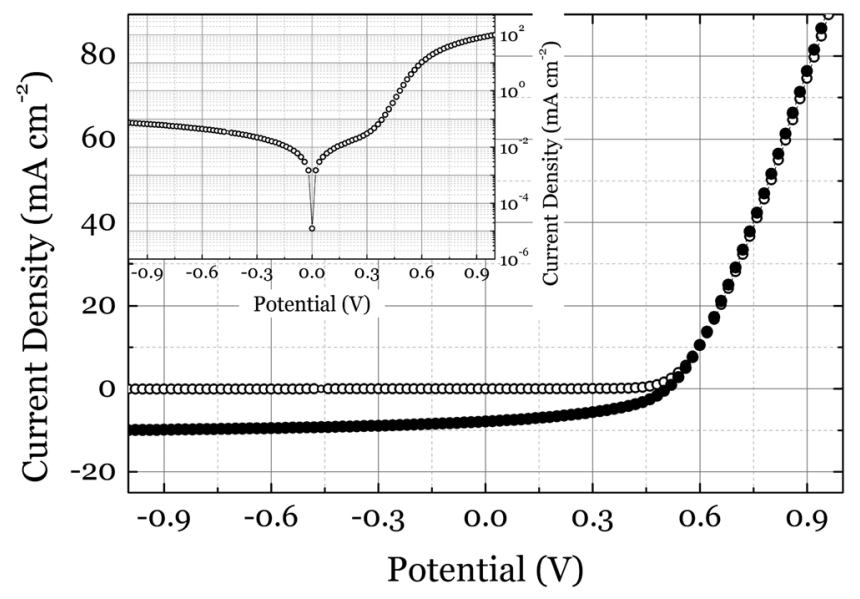

FIG. 2. Current-voltage characteristics measured in dark ( 0 ) and under illumination $(\bullet) 1.5 \mathrm{AM} 100 \mathrm{~mW} / \mathrm{cm}^{2}$ with respect to a reference silicon diode. The inset shows the dark IV curves in semi-log scale.
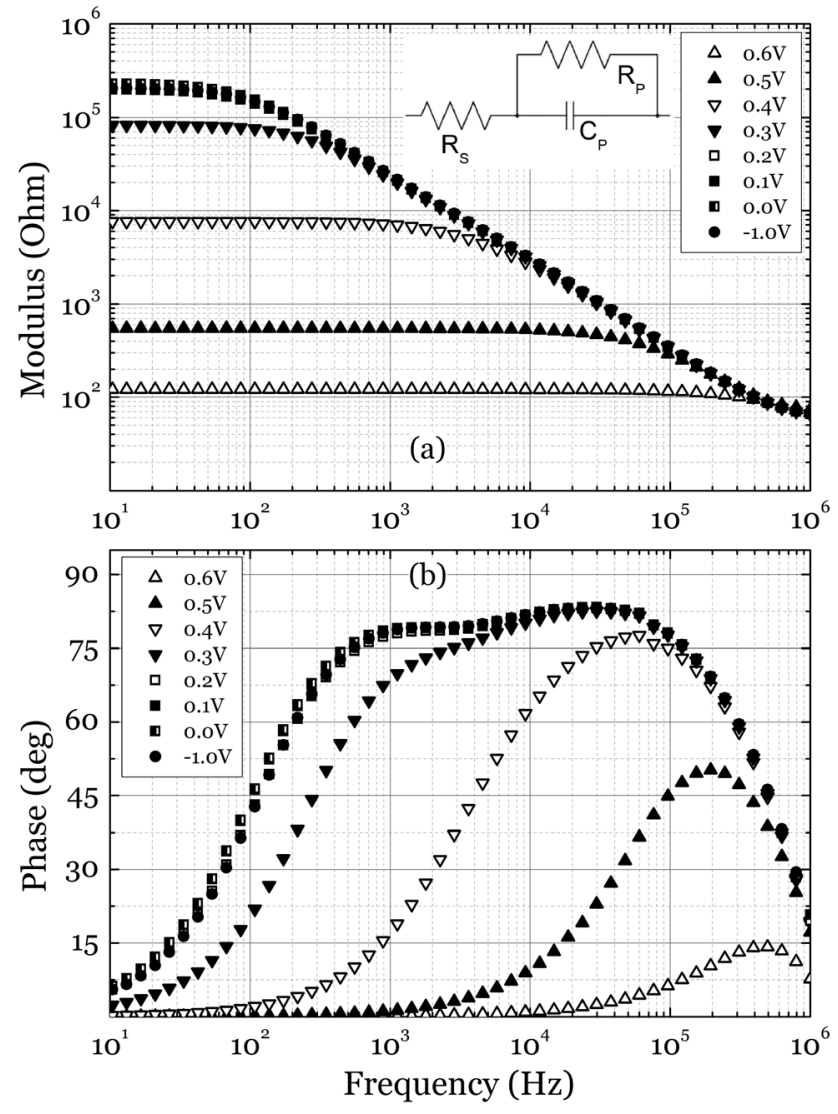

FIG. 3. Modulus (a) and phase (b) spectra of the device impedance for different applied bias potentials. In the modulus spectra, the high frequency plateau is associated with the series resistance. At low frequencies, the plateau is correlated with the parallel resistance and is strongly voltagedependent. In the phase spectra, the trap states' peak is visible at frequencies around $1 \mathrm{kHz}$ for reverse and low forward bias.

The bias voltage is considered positive when negative potential is applied to aluminum contact and positive to ITO.

In the modulus spectrum (Fig. 3(a)), we observe two plateaus in the low- and high-frequency regions. In the medium frequency range, the modulus changes as $1 /\left(\omega C_{\mathrm{P}}\right)$, as a result of the device capacitance. This behavior can be explained with a simple equivalent circuit composed by one $\mathrm{RC}$ parallel circuit plus a series resistance (inset of Fig. 3(a)). At high frequencies, the modulus plateau is not clearly visible. It is more evident from the phase which decreases with increasing frequency, which is a sign of a resistive contribution (Fig. 3(b)). The absolute value of the impedance at the higher frequencies corresponds to the series resistance, which takes into account the resistive contribution of the contact lines and the doped transport layers. As expected, it is voltage independent and is in the range of 60-70 $\Omega$.

The plateau at low frequencies is composed of the combination of the parallel and series resistances. This changes significantly with the applied voltage and is directly correlated to the slope in the steady-state current-voltage characteristic. The physical meaning of this resistance lies in the non-ohmic-injection from the contacts and in the intensity of the recombination process in the intrinsic blend layer.

The charge carriers injected from the doped transport layers need to recombine in the active layer or at the 
interface with the doped layers to support the current flowing in the device. ${ }^{25}$ These processes are voltage and current dependent; therefore, the parallel resistance changes with the applied bias. For the intermediate frequencies, the parallel capacitance is contributing to the impedance with the characteristic slope of $1 /\left(\omega C_{\mathrm{P}}\right)$. This region is analyzed in more detail in the following.

Figure 3(b) shows the phase spectrum of the measured impedance. For bias voltages between $0.4 \mathrm{~V}$ and $0.6 \mathrm{~V}$, a single peak is visible, as expected in the presence of a single $\mathrm{RC}$ plus a series resistance. On the other hand, a second peak around $1 \mathrm{kHz}$ becomes visible for bias voltages lower than $0.3 \mathrm{~V}$ or negative. This is the effect of trap states that are starting to respond when the modulation signal is slowly enough to be followed by the trapping-detrapping process.

The presence of a significant current flowing through the device $(0.4-0.6 \mathrm{~V})$ suppresses the traps response, because the parallel resistance is very small and the device behavior turns to purely resistive. In this condition, it is not possible to correctly measure the phase shift of the current signal and the imaginary part of the impedance in the low frequency region. In addition, the strong charge carrier injection fills the trap states, preventing the small signal to activate a trapping-detrapping process, since the trap occupancy function is strongly affected by charge injection and, subsequently, also the process dynamics. ${ }^{26}$

In summary, from the analysis of the measured impedance spectra, we find that, for positive bias, the equivalent circuit for a small-molecule organic solar cell is composed by an RC parallel circuit plus a series resistance, as shown in the inset of Fig. 3(a). For negative and small positive bias, the response of trap states induces an additional capacitive contribution that is clearly visible as a second peak in the low frequency region of the phase spectrum of Fig. 3(b).

The change in occupation of these trap states can be attributed to a charge transfer or to a partial penetration of dopant molecules in the intrinsic layer during the evaporation. As we have already shown in a previous work, ${ }^{15}$ for totally intrinsic devices, the traps respond only in the presence of illumination and photo-generated charges. Moreover, the possibility to activate these states by illumination proves that they are located in the bulk-heterojunction active layer.

In the case discussed in this work, the traps are visible in the complex capacitance spectra also in the dark. From this observation, we propose that doped layers introduce free charges in the blend (either by charge transfer or dopant diffusion) and activate the trap states. In Sec. III C, we focus on modeling this contribution by using an equivalent circuit, giving it a well-defined physical meaning.

\section{Equivalent circuit of distributed trap states}

To clearly show the contribution of trap states, the results are presented also in terms of complex capacitance. This quantity is derived from the measured impedance as: $C(\omega)=Y(\omega) /(j \omega) . Y(\omega)$ is the complex admittance, defined as the inverse of the impedance $Z(\omega)$, and $\omega$ is the angular frequency. With this notation, the real part of the complex capacitance describes the capacitive response of the device.
At the same time, the imaginary part is related to the dissipation of energy within the medium or, in terms of electrical quantities, it is the device conductance multiplied by the angular frequency. ${ }^{24}$

It has been shown that, in order to characterize the trap states, it is necessary to avoid a significant current injection from the contacts, which can affect the boundary conditions necessary for a proper characterization of the traps' dynamics. ${ }^{27}$ In the forward region, the device behavior is strongly conductive and the capacitive contribution of traps cannot be determined with precision. On the contrary, in reverse bias, with low dark currents, the device response is mostly capacitive and the trap contribution can be clearly distinguished. We therefore focus on the reverse bias condition of our device. The trap states' contribution is then evaluated, observing the real part of the complex capacitance.

The characterization of trap states for inorganic semiconductors has been well studied, and an equivalent circuit for the trap impedance was presented by Losee. ${ }^{19}$ They reported that the effect of a single trap state, not acting as a recombination center, is equivalent to a $\mathrm{RC}$ series circuit. In that model, the resistance took into account the energy loss that occurs in the passage of the electrons from the transport level (lowest unoccupied molecular orbital (LUMO) - in the case of organic semiconductors) to the intra-gap state, and the capacitance represented the occupied state of the trap, which produces an additional charge accumulation and influences the electric field.

Even if this approach can describe the impedance in conventional inorganic semiconductor devices, it is not sufficient for organic semiconductors, where the intra-gap states cannot be described as discrete levels, but by a certain distribution in energy. In a general case of a trap state distribution function $g(E)$, it is possible, under the assumption of Boltzmann statistics, to calculate the trap capacitance by integrating their energy distribution, ${ }^{20}$

$$
C(\omega)=\frac{q^{2}}{k_{B} T} \int_{E_{v}}^{E_{c}} \frac{g(E) f_{t}(E)\left[1-f_{t}(E)\right]}{1+j \omega / \omega_{t}} d E,
$$

where $\omega_{\mathrm{t}}$ is the trap frequency, $f_{\mathrm{t}}(E)$ is the states' distribution function, and $g(E)$ is the density of states. This is a general solution for the organic semiconductor case, but needs further simplifications in order to be applied to real devices. Moreover, the solution of the integral requires the knowledge of the Fermi level position in the device, which, under operating conditions, is not available in most cases. Thus, we propose a generalization of the Losee model and an equivalent circuit to describe energetically distributed trap states.

First, we would like to comment about the use of the constant phase elements (CPEs) in the fitting procedure. One could introduce CPEs in the Losee model in place of standard capacitors and describe the distribution of trap response times. In this way, it is possible to obtain results similar to the equivalent circuit proposed here, but the physical meaning of the exponent factor in the CPE expression is not clear and the obtained values can hardly be used for a quantitative analysis. The possibility to use a combination of ideal components instead of a purely mathematical element produces 
great advantages in the physical explanation of the results obtained.

In principle, the trap capacitance of a distribution of states can be obtained by summing the single contribution of every single intra-gap state. In terms of an equivalent circuit, this consideration can be translated to a large number of RC series circuits, each one modeling one trap state. However, it is not feasible to use an equivalent circuit with an almost infinite number of parameters. Thus, a simplification is necessary to obtain an applicable equivalent circuit. For this, if we assume an uniform energetic distribution of trap states, it is possible to use the same capacitance for every trap state and the resistor increases linearly with the depth of the trap state in energy.

The circuit element that corresponds to this definition is an infinite repetition of the same RC element, each one ideally representing a single trap state at a different energy level and with a specific trapping-detrapping time, as shown in the inset of Fig. 4. The elements are arranged in such a way that the subsequent capacitance element is connected to the contact by an increasing number of elementary resistors, characterized by a different time constant (e.g., RC, 2RC, $3 R C . .$.$) . It is important to observe that no direct resistive$ path is present between the two contacts, which means that the traps are considered not to act as recombination centers, therefore, not directly contributing to the current.

Figure 4 shows the spectra of the real and imaginary part of the measured complex capacitance at $0.0 \mathrm{~V}$ and $-1.0 \mathrm{~V}$ bias voltage. No significant changes are visible applying different reverse potentials. The spectra can be divided

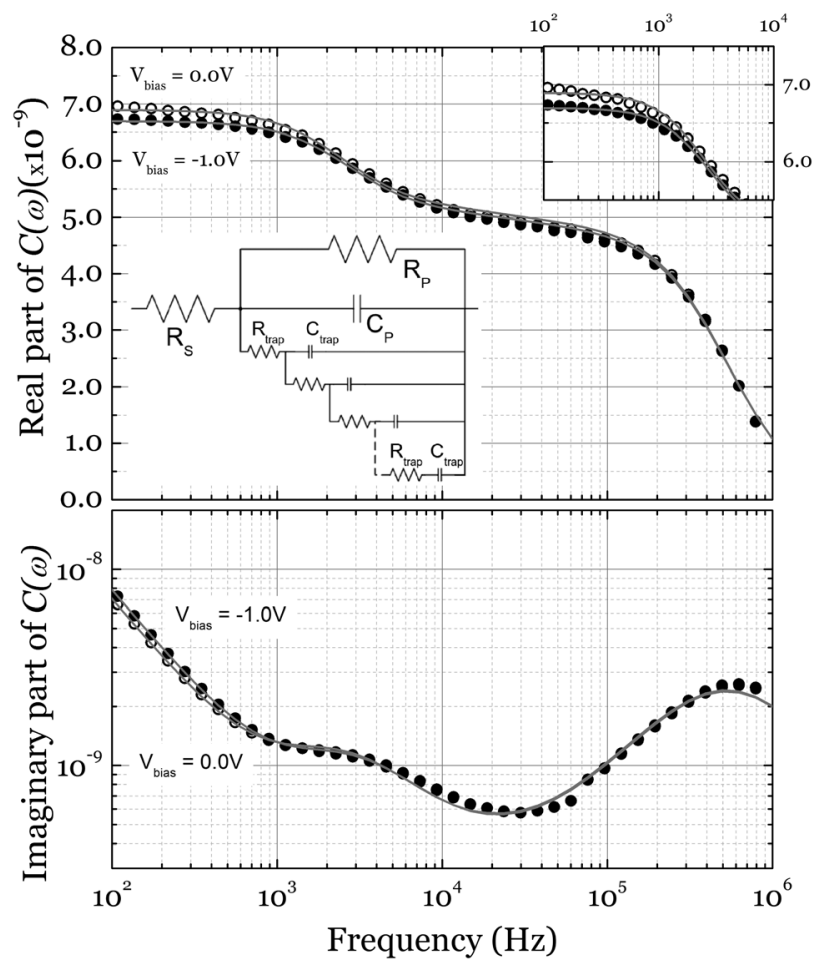

FIG. 4. Real and imaginary part of the complex capacitance at $0.0 \mathrm{~V}$ and $-1.0 \mathrm{~V}$ bias potential. The small changes of the impedance with the reverse voltage can be better observed in the inset. The spectra are fitted using the circuit shown in the inset, and the simulated curves are presented as continuous lines. in three different regions: starting from high frequencies, above $100 \mathrm{kHz}$, we observe a decrease in the real part, due to the increasing contribution of the series resistance to the overall impedance, as already observed in the modulus spectra of Fig. 3(a).

Between $10 \mathrm{kHz}$ and $100 \mathrm{kHz}$, we observe a plateau in the real part, which can be directly correlated to the value of the parallel capacitance $C_{\mathrm{P}}$ already discussed previously. The presence of doped layers avoids the formation of depletion layers at the metal/organic interface. As a consequence, this plateau is voltage independent and equals the geometrical capacitance of the intrinsic layer $\left(C_{\text {geo }}=4.7 \mathrm{nF}\right.$ with $\varepsilon=4$ ), as can be observed in the values reported in Table I.

The plateau is not as perfectly flat as it should be for an ideal capacitor. The reason is the contribution of shallow trap states, which contribute to the transport and do not give a purely capacitive contribution, but still can temporarily store charge carriers. This intermediate behavior of trap states closer to the transport level can be successfully modeled by the proposed equivalent circuit. For frequencies lower than $10 \mathrm{kHz}$, the additional capacitive contribution is attributed to the trap states. These states start to respond from frequencies lower than $10 \mathrm{kHz}$, and they are all responding when the real complex capacitance reaches the low frequencies' plateau.

The calculated spectra obtained from fitting with the proposed equivalent circuit are shown as continuous lines and can reproduce the measured data well. It is important to observe that we have employed only ideal capacitors in the fitting procedure, and the traps contribution is implemented with a transmission line. Here, we use the fitting software Zview ${ }^{\circledR}$, implementing the transmission line response by a large repetition of discrete elements.

The discretization of such a distributed circuit element does not introduce significant deviations from the ideal transmission line response for single element repetitions higher than $100 .{ }^{28}$ We report in Table I the elementary value multiplied by the number of repetitions, here, 1000 .

The complex capacitance spectra does not change significantly with the reverse voltage, as shown in the inset of Fig. 4 and the values obtained from the fitting in Table I. In the medium frequency range, the response is dominated by the capacitance of the blend layer that is fully depleted and the capacitance equals the geometrical value, which is voltage independent.

We observe that the traps' capacitance is voltage independent as well. As explained by Cohen et al., ${ }^{27}$ the frequency variation corresponds to a scan in the energy of the distribution of states, while the voltage scans the position.

TABLE I. Summary of the results obtained from fitting the measured data with the equivalent circuit presented in Fig. 4. The fitting errors are below $\pm 1 \%$ for the $R_{\mathrm{S}}$, the $R_{\mathrm{P}}$, and the $C_{\mathrm{P}}$. The $R_{\text {traps }}$ has an error of $\pm 6 \%$ and the $C_{\text {traps }}$ of $\pm 2.5 \%$.

\begin{tabular}{lccccc}
\hline \hline Bias $(\mathrm{V})$ & $R_{\mathrm{S}}(\Omega)$ & $R_{\mathrm{P}}(\mathrm{k} \Omega)$ & $C_{\mathrm{P}}(\mathrm{nF})$ & $R_{\text {traps }}(\mathrm{k} \Omega)$ & $C_{\text {traps }}(\mathrm{nF})$ \\
\hline 0.0 & 61.15 & 227.21 & 4.79 & 73.85 & 2.11 \\
-0.5 & 61.43 & 217.59 & 4.75 & 73.02 & 2.02 \\
-1.0 & 61.49 & 204.34 & 4.73 & 71.77 & 1.97 \\
\hline \hline
\end{tabular}


The capacitance at low frequencies is composed of a series capacitance from the deep depletion region (DDR) and the semiconductor capacitance (SC). ${ }^{27,29}$ The capacitance arising from the DDR is dependent on the applied bias. The SC, on the other hand, is voltage independent, as it is only given by the number of traps responding at that frequency. Thus, this is frequency dependent. For low reverse bias, as in the present case, the capacitance is dominated by the semiconductor capacitance and, hence, is frequency dependent, but voltage independent.

The frequency dependence is given by the specific dynamic response of the trap states that are not able to respond to signals faster than the trapping-detrapping characteristic time. At low frequencies (below $10 \mathrm{kHz}$ ), the trap states are able to follow the applied modulation and the additional capacitance contribution is observed. The elementary capacitance value, $C_{\text {traps }}$, can be used to estimate the density of states above $E_{F}$, assuming a constant trap density, $g_{0}$. In this case, $C_{\text {traps }}=A \sqrt{q g_{0} \varepsilon}, 27,29,30$ and from the value obtained at $0.0 \mathrm{~V}$ bias potential, we estimate $g_{0}$ to be around $1.9 \pm 0.6 \times 10^{16} \mathrm{~cm}^{-3} \mathrm{eV}^{-1}$, which is a reasonable value for organic semiconductors. ${ }^{16}$

\section{CONCLUSIONS}

The approach and model we have presented for describing the capacitive response of trap states in organic semiconductors has the advantage of simple application and physical interpretation. The use of a transmission line introduces some simplifications to the general model of Eq. (1). This simplification limits the possibility to obtain direct information about the traps' distribution function. However, by using the same values for every RC element, we are able to reduce the fitting parameters to a manageable amount. In conclusion, the equivalent circuit proposed is able to model the capacitive contribution of trap states in organic semiconductors. This equivalent circuit only requires a small number of parameters, thereby reducing fitting errors. The introduction of a distributed element to model the trap capacitance has its physical justification in the energetic distribution of trap states in an organic semiconductor. The experimental results can be understood with the equivalent circuit proposed, and the trap state contribution can be evaluated. From the transmission line elements, we estimate the density of trap states to be around $1.9 \pm 0.6 \times 10^{16} \mathrm{~cm}^{-3} \mathrm{eV}^{-1}$ in a $\mathrm{ZnPc}: \mathrm{C} 60$ bulk heterojunction.

\section{ACKNOWLEDGMENTS}

The authors would like to thank the BMBF for funding in the framework of the OPEG project (13N9720) and Novaled AG for dopant supply.

${ }^{1}$ C. Deibel and V. Dyakonov, Rep. Prog. Phys. 73, 96401 (2010).

${ }^{2}$ M. K. Riede, R. Schueppel, K. Schulze, D. Wynands, R. Timmreck, C. Uhrich, A. Petrich, M. Pfeiffer, E. Brier, E. Reinold, P. Baeuerle, and K. Leo, Proc. SPIE 7002, 70020 G (2008).

${ }^{3}$ See www.heliatek.de for record cell efficiency $9.8 \%$.

${ }^{4}$ See www.konarka.com for record cell efficiency $9.0 \%$.

${ }^{5}$ See www.solarmer.com for record cell efficiency $8.1 \%$.

${ }^{6}$ M. Riede, T. Mueller, W. Tress, R. Schueppel, and K. Leo, Nanotechnology 19, 424001 (2008).

${ }^{7}$ R. Schueppel, R. Timmreck, N. Allinger, T. Mueller, M. Furno, C. Uhrich, K. Leo, and M. Riede, J. Appl. Phys. 107, 44503 (2010).

${ }^{8}$ J. Shao and G. Wright, Solid-State Electron. 3, 291 (1961).

${ }^{9}$ T. Walter, R. Herberholz, C. Muller, and H. W. Schock, J. Appl. Phys. 80, 4411 (1996).

${ }^{10}$ N. Nguyen, M. Schmeits, and H. Loebl, Phys. Rev. B 75, 75307 (2007).

${ }^{11}$ H. Martens, H. Brom, and P. Blom, Phys. Rev. B 60, R8489 (1999).

${ }^{12}$ S. W. Tsang, S. K. So, and J. B. Xu, J. Appl. Phys. 99, 13706 (2006).

${ }^{13}$ J. M. Montero, J. Bisquert, G. Garcia-Belmonte, E. M. Barea, and H. J. Bolink, Org. Electron. 10, 305 (2009).

${ }^{14}$ G. Garcia-Belmonte and J. Bisquert, Appl. Phys. Lett. 96, 113301 (2010).

${ }^{15}$ D. Ray, L. Burtone, K. Leo, and M. Riede, Phys. Rev. B 82, 125204 (2010).

${ }^{16}$ P. P. Boix, G. Garcia-Belmonte, U. Munecas, M. Neophytou, C. Waldauf, and R. Pacios, Appl. Phys. Lett. 95, 233302 (2009).

${ }^{17}$ M. M. Mandoc, F. B. Kooistra, J. C. Hummelen, B. de Boer, and P. W. M. Blom, Appl. Phys. Lett. 91, 263505 (2007).

${ }^{18}$ G. I. Roberts, J. Appl. Phys. 41, 1767 (1970).

${ }^{19}$ D. L. Losee, J. Appl. Phys. 46, 2204 (1975).

${ }^{20}$ J. Bisquert, Phys. Rev. B 77, 235203 (2008).

${ }^{21}$ K. Walzer, B. Maennig, M. Pfeiffer, and K. Leo, Chem. Rev. 107, 1233 (2007).

${ }^{22}$ R. Meerheim, S. Olthof, M. Hermenau, S. Scholz, A. Petrich, N. Tessler, O. Solomeshch, B. Lussem, M. Riede, and K. Leo, J. Appl. Phys. 109, 103102 (2011).

${ }^{23}$ F. A. Cotton, Inorg. Chem. Commun. 6, 121 (2003).

${ }^{24}$ E. Barsoukov and J. Macdonald, Impedance Spectroscopy: Theory, Experiment, and Applications, 2nd ed. (Wiley, 2005).

${ }^{25} \mathrm{P}$. Wuerfel, Physics of Solar Cells: From Basic Principles to Advanced Concepts (Wiley-VCH, 2005)

${ }^{26}$ Y. Zhang, B. de Boer, and P. W. M. Blom, Phys. Rev. B 81, 85201 (2010).

${ }^{27}$ J. Cohen and D. Lang, Phys. Rev. B 25, 5321 (1982).

${ }^{28}$ See ZView TM software on www.scribner.com.

${ }^{29}$ D. Ray and K. L. Narasimhan, J. Appl. Phys. 103, 93711 (2008).

${ }^{30}$ D. K. Sharma, K. L. Narasimhan, S. Kumar, B. M. Arora, W. Paul, and W. A. Turner, J. Appl. Phys. 65, 1996 (1989). 